\title{
Nuestra experiencia con minicintas (TVT Secur y MiniArc) en la cirugía de la incontinencia urinaria de esfuerzo
}

\author{
Jiménez Calvo J, Hualde Alfaro A, Raigoso Ortega O, Cebrian Lostal JL, Alvarez Bandres S, \\ Jiménez Parra J, Montesino Semper M, Santiago Gonzalez de Garibay A.
}

\author{
Servicio de Urología. Hospital Virgen del Camino. Pamplona. Navarra.
}

Actas Urol Esp. 2008;32(10):1013-1018

\section{RESUMEN}

NUESTRA EXPERIENCIA CON MINICINTAS (TVT SECUR Y MINIARC) EN LA CIRUGIA DE LA INCONTINENCIA URINARIA DE ESFUERZO

Objetivos: El objetivo de esta publicación es describir la técnica quirúrgica, evaluar las complicaciones y los resultados a corto plazo de las cintas TVT secur y MiniArc.

Material y Método: entre Octubre de 2006 y Agosto 2007 se realizó corrección quirúrgica con TVT SecurTM, Women's Health \& Urology, Ethicon, Johnson \& Johnson, colocando la cinta en forma de hamaca, a 51 pacientes,38 de ellas con incontinencia de esfuerzo pura y 13 con incontinencia mixta y con una edad media de 57 años.Entre Septiembre 2007 y Febrero de 2008 se intervino con cinta AMS MiniarcTM swing system, colocada en hamaca, a 41 pacientes, 33 pacientes con incontinencia de esfuerzo pura y 8 con incontinencia mixta, con un edad media de 58 años. Todos los procedimientos se realizan con sedoanalgesia y en régimen de Cirugía Mayor Ambulatoria. Las pacientes fueron controladas en consultas externas al mes, 3 meses y al año. Se les realizó historia clínica y cuestionario ICIQ- SF al que le añadimos una pregunta para cuantificar el grado de satisfacción, así como exploración física. Comparamos los resultados de ambas técnicas y realizamos estudio estadístico mediante test de Student. [Análisis con el programa informático SPSS (V14.0)].

Resultados: La mediana de seguimiento en el grupo TVT secur fue de 328 días (rango 163-522 días) y en el grupo MiniArc de 101 días (rango 41-209 días). De las 92 paciente intervenidas, 51 con TVT secur y 41 con MiniArc solo registramos una complicación quirúrgica con TVT secur que fue una perforación vesical. Considerando como curación objetiva que la paciente en la exploración física realizada en consulta con vejiga llena presente un test de esfuerzo negativo. En el grupo TVT secur el 80,4\% de las pacientes están curadas y en el grupo de MiniArc el 90,2\% no existiendo diferencias significativas entre ambos grupos( p 0,095). Para evaluar la curación subjetiva utilizamos el test ICIQ-SF y la pregunta de satisfacción y observamos como, en el control al mes y tercer mes no existen diferencias significativas, estando el 90\% de las pacientes satisfechas. En el control anual, sólo realizado en el grupo TVT secur, el $80 \%$ de las pacientes se encuentran muy satisfechas.

Conclusión: Estas nuevas cintas presentan un número menor de complicaciones permiten la posibilidad de colocación con anestesia local pero todavía son necesarios estudios aleatorizados con un mayor seguimiento.

Palabras clave: Incontinencia. TVT secur. MiniArc.

\section{ABSTRACT}

OUR EXPERIENCE WITH MINI TAPES (TVT SECUR AND MINIARC) IN THE SURGERY FOR STRESS URINARY INCONTINENCE

Background: The purpose of this publication is to describe the surgical technique, assess complications and short-term results of TVT secur and MiniArc tapes.

Materials and Methods: From October 2006 to August 2007 it was carried out the surgical correction with TVT SecurTM, Women's Health \& Urology, Ethicon, Johnson \& Johnson, placing the tape as a hammock, to 51 patients, 38 of them with pure stress incontinence and 13 with mixed incontinence and with an average age of 57 years. From September 2007 to February 200841 patients, 33 of them with pure stress incontinence and 8 with mixed incontinence, with an average of age of 58 years were operated with AMS MiniarcTM swing system tape, posted on hammock. All procedures were performed with sedoanalgesia and Ambulatory Surgery regime. Patients were monitored in outpatient visits one month, 3 months and one year after surgical procedure .Medical history and questionnaire and ICIQ-SF, to which we added a question to quantify the degree of satisfaction, as well as physical examination, were done. We compared the results of both technical procedures and statistical survey was conducted by Student test. [Analysis with SPSS software (V14.0)].

Results: The median follow-up in TVT secur group was 328 days (range 163-522 days) and 101 days (range 41-209 days) in the MiniArc group. We only had one (TVT secur group) surgical complication in all the series (92 patients) being a bladder perforation. Taking into account that we read a negative test effort as an objective cure in the TVT secur group, $80.4 \%$ patients are cured and $90.2 \%$ in the MiniArc group without significant difference between both groups ( $\mathrm{p}$ 0095). To assess the subjective healing we utilized the ICIQ-SF test and the satisfaction extra-question and we noted that there is no significant difference between the first and third month controls. (90\% of patients satisfied). $80 \%$ of patients were completely satisfied in the first year control that was only performed to TVT secur group.

Conclusion: These new tapes show fewer complications that the TVT-O and TOT tapes and allow the possibility of placement with local anesthesia but further studies are needed.

Keyword: Incontinence. TVT secur. MiniArc. 
$\mathrm{D}$ esde la llegada de la TVT en el año 1996 han ido apareciendo diferentes técnicas mínimamente invasivas para la cirugía de la incontinencia urinaria de esfuerzo, todas ellas basadas en las teorías integrales. En octubre de 2006 aparece TVT Secur ${ }^{\mathrm{TM}}$, Women's Health \& Urology, Ethicon, Johnson \& Johnson, cinta de menor longitud y en el 2007 las AMS MiniArc ${ }^{\mathrm{TM}}$ swing system y Needeless ${ }^{\mathrm{TM}}$. Estas cintas pueden colocarse en forma de "V" (como en la TOT/TVT-O) quedando la cinta anclada en el músculo obturador interno o en forma de "U" (como en la TVT) quedando la cinta en el espacio retropúbico.

El objetivo de esta comunicación es analizar nuestra experiencia de forma retrospectiva con la utilización de estas minicintas y comparar las técnicas en cuanto a resultados y complicaciones.

\section{MATERIAL Y MÉTODO}

Entre Octubre de 2006 y Agosto de 2007 hemos intervenido a 51 pacientes, colocándoles TVT secur en forma "V". La edad mediana de 57 años con un rango entre 38 y 81 años. De las 51 pacientes 38 tenían incontinencia urinaria de esfuerzo y 13 incontinencia urinaria mixta.

Entre Agosto de 2007 y Febrero de 2008 hemos intervenido a 41 pacientes, colocándoles MiniArc en forma de "V". La edad mediana es de 58 años con un rango entre 37 y 82 años. De las 41 pacientes 33 presentan incontinencia urinaria de esfuerzo pura y 8 incontinencia urinaria mixta.

A todas ellas se les realiza como parte del estudio preoperatorio una historia clínica, exploración física y en el caso de incontinencia urinaria mixta se le solicita urodinámica. Dentro de la historia clínica las pacientes complementen el cuestionario ICIQ-SF.

La mediana de seguimiento en el grupo TVT secur fue de 328 días (rango 163-522 días) y en el grupo MiniArc de 101 días (rango 41-209 días).

Consideramos como curación objetiva que la paciente en la exploración física realizada en consulta con vejiga llena presente un test de esfuerzo negativo y para evaluar la curación subjetiva evaluamos los resultados del cuestionario ICIQ-SF.

Esta cirugía la realizamos con sedoanalgesia y en régimen de cirugía mayor ambulatoria. Solicitamos como único estudio preoperatorio una analítica con coagulación.

\section{Técnica quirúrgica y anestésica}

Previamente a la colocación de la paciente en posición ginecológica en $90^{\circ}$ de flexión muslo-abdomen se administra profilaxis antibiótica con cefazolina 2 gramos y se le monitoriza.

Se administra por vía iv 1 miligramo de midazolan y se comienza con infusión de $50 \mathrm{mg}$ de meperidina diluidos en $100 \mathrm{cc}$ de suero fisiológico. A nivel de introito vaginal se aplica aerosol de Xilocaina.

Comprobamos el escape con maniobra de Valsalva previamente a la cirugía

Tras vaciar la vejiga mezclamos 3 ampollas de lidocaína con 30 cc de suero fisiológico que inyectamos en cara anterior y con aguja epidural infiltramos la trayectoria de la cinta hasta músculo obturador interno.

Una vez inyectada la anestesia local realizamos la incisión vaginal longitudinal a $1 \mathrm{~cm}$ del meato y procedemos a realizar la disección parauretral con la tijera y colocación de la cinta (TVT secur/MiniArc) en forma de hamaca, anclando las cintas en el músculo obturador interno.

Seguidamente llenamos la vejiga y retiramos la sonda vesical, ajustando la tensión de la cinta hasta que comprobamos que no existe escape con la maniobra de Valsalva. Vaciamos la vejiga y suturamos la incisión vaginal con puntos de Vycril 3/0.

Sin sonda vesical ni taponamiento vaginal la paciente vuelve a la Unidad de Cirugía Mayor Ambulatoria donde se le medirá 2 residuos postmiccionales. Si éstos son mayores de $100 \mathrm{cc}$ se le da el alta con sonda vesical.

Las pacientes fueron controladas en consultas externas al mes, 3 meses y al año. Se les realiza historia clínica y cuestionario ICIQ- SF al que le añadimos una pregunta para cuantificar el grado de satisfacción (Fig. 1) así como exploración física.

Para evaluar la curación utilizamos como criterio objetivo que la paciente en la exploración física realizada en consulta con vejiga llena presente un test de esfuerzo negativo y para evaluar la subjetiva los resultados del test ICIQ-SF junto con la pregunta de satisfacción. Comparamos ambas técnicas y realizamos estudio estadístico mediante test de Student. [Análisis con el programa informático SPSS (V14.0)].

\section{RESULTADOS}

De las 92 paciente intervenidas, 51 con TVT secur y 41 con MiniArc sólo registramos una complicación quirúrgica con TVT secur que fue una perforación vesical. 


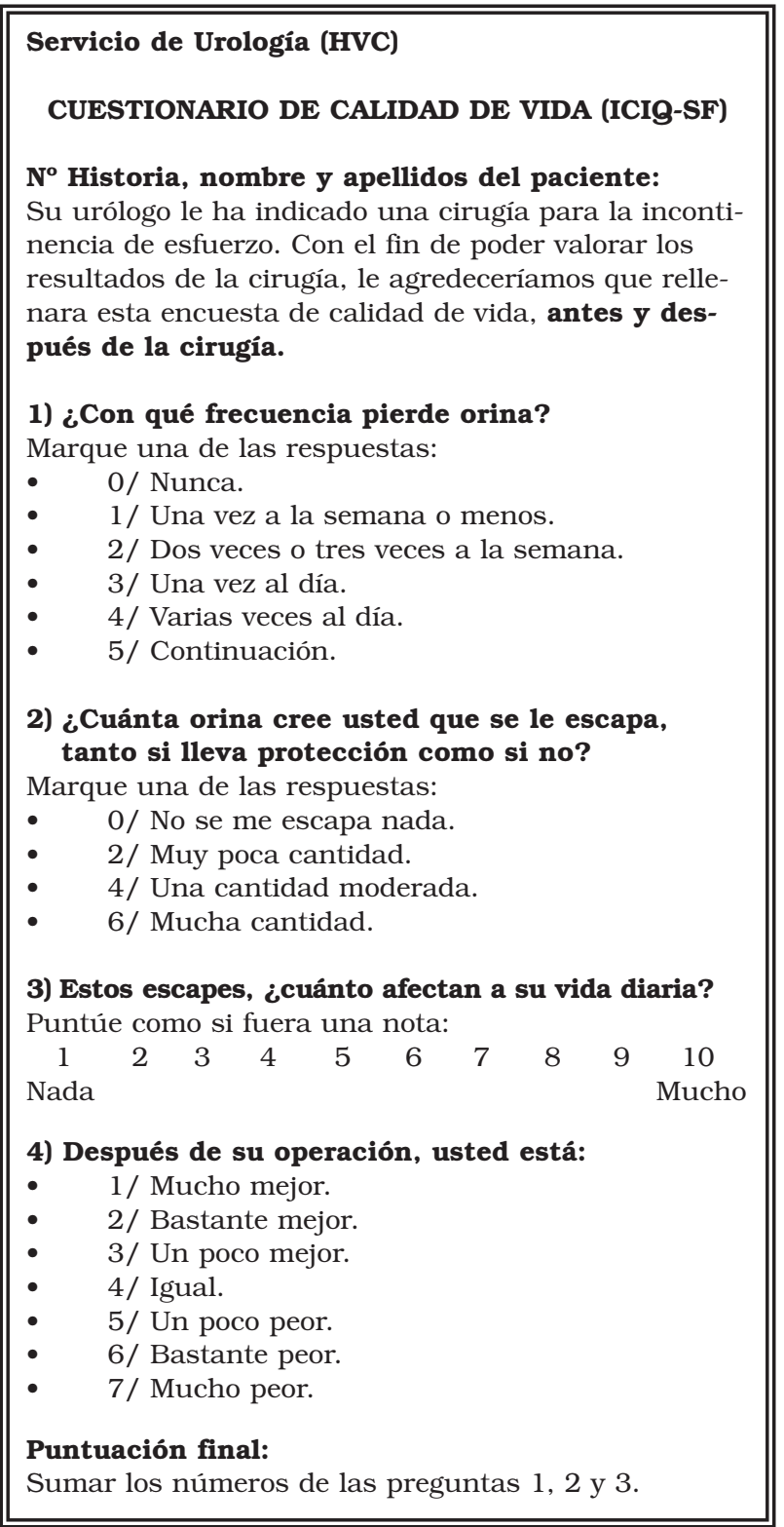

FIGURA 1. ICIQ-SF y pregunta de satisfacción.

Como complicaciones postoperatoria en el grupo TVT secur se presentaron: portadoras de sonda vesical de más de un día pero menos de 7 días por dificultades miccionales en 2 pacientes, 1 paciente necesitó el corte de la cinta por obstrucción, 2 cirugías por extrusión de la cinta y en 5 pacientes apareció urgencia miccional de novo

Como complicaciones postoperatoria en el grupo MiniArc se presentaron: portadoras de sonda vesical de más de un día pero menos de 7 por dificultades miccionales en 3 pacientes; 1 paciente necesitó corte de la cinta por obstrucción y en 5 pacientes apareció urgencia miccional de novo.
La mediana del ICIQ-SF preoperatorio del grupo TVT secur fue de 15,79 desviación estándar de 3,03 y del grupo MiniArc de 15,05 desviación estándar de 2,46 no existiendo diferencias significativas entre ambos grupos (p 0,242) (Fig. 2).

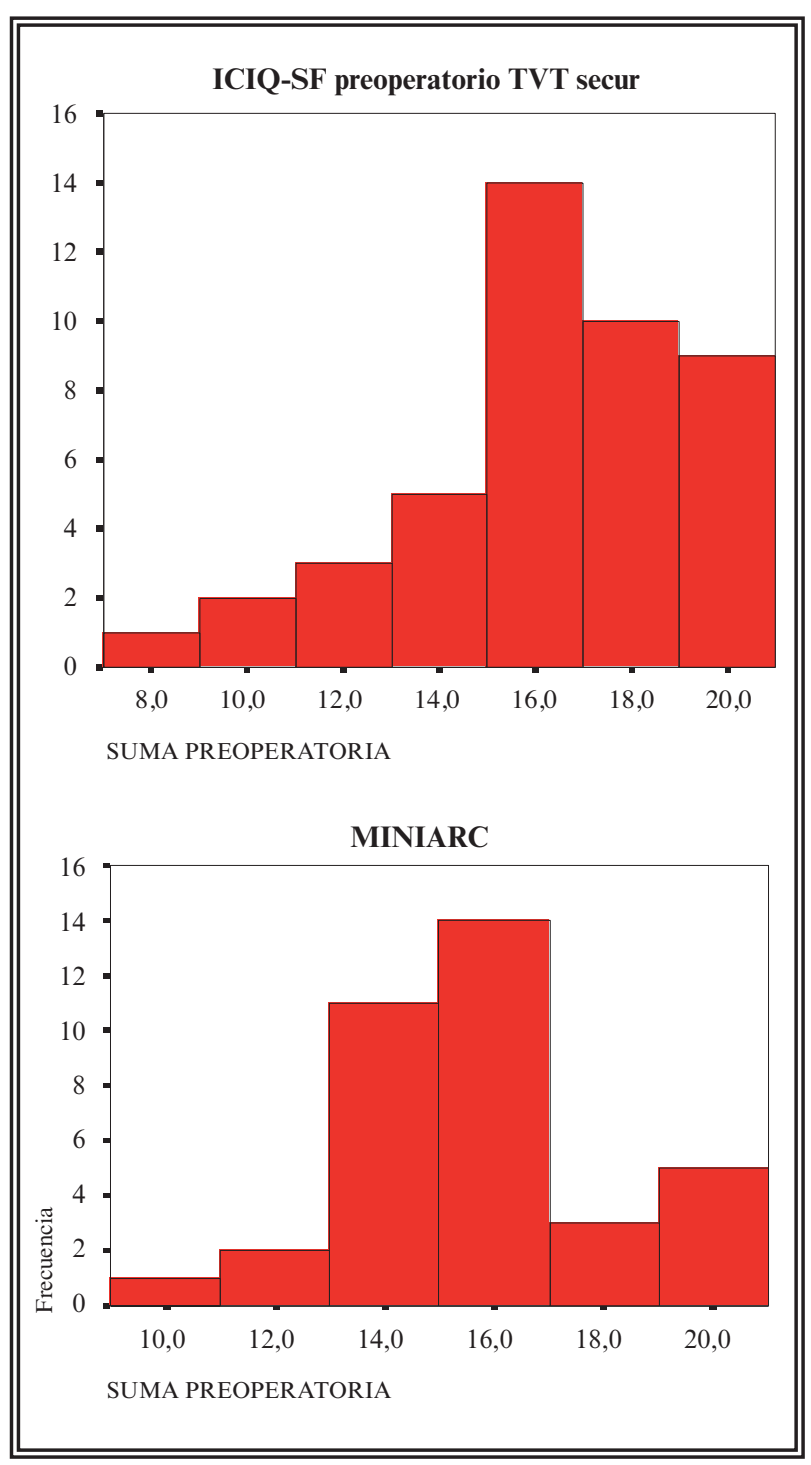

FIGURA 2. ICIQ-SF preoperatorio.

Considerando como curación objetiva que la paciente en la exploración física realizada en consulta con vejiga llena presente un test de esfuerzo negativo, en el grupo TVT secur, el $80,4 \%$ de las pacientes están curadas y en el grupo de MiniArc el $90,2 \%$ no existiendo diferencias significativas entre ambos grupos (p 0,095).

Para evaluar la curación subjetiva utilizamos el test ICIQ-SF ${ }^{14}$ y la pregunta de satisfacción y observamos 
como en el control del mes la suma para el grupo TVT secur la media de la suma es de 1,5 (desviación de $3,56)$ y en cuanto a la pregunta de satisfacción observamos como el 94,6\% de las pacientes están bastante o muy satisfechas. Para el grupo MiniArc vemos que la media de la suma para el ICIQ-SF es de 1,45 (desviación de 3,56) y en cuanto a la pregunta de satisfacción el 94\% de ellas están bastante o muy satisfechas. Si comparamos ambos grupos no existen diferencias significativas en ninguno de los aspectos analizados. Si observamos los resultados de la pregunta 1 del test ICIQ-SF que nos indica la frecuencia de perdidas de orina advertimos como en el grupo TVT secur como no existen perdidas o sólo ocurre una vez a la semana coincidiendo con algún esfuerzo ocurre en el 84,6\% y en el grupo MiniArc en el 83,8\% (Fig. 3).

Los resultados a los 3 meses se repiten no existiendo diferencias significativas en ambos grupos tanto en el cuestionario ICIQ-SF (grupo TVT secur media 2,6 con desviación de 4,74; grupo MiniArc media 1,4 con desviación 3,52 ) como en la pregunta de satisfacción ya que el $90,9 \%$ de las pacientes del grupo TVT secur y el 88,6\% del grupo MiniArc se encuentran bastante o muy satisfechas (Fig. 4).

Los resultados al año (sólo evaluables con TVT secur) vemos como la media de ICIQ-SF es de 3,3 con una desviación de 5,96 y el grado de satisfacción (Bastante/mucho mejor) es del 80,4\% (Fig. 5).

\section{DISCUSIÓN}

La primera generación de cintas suburetrales fue la TVT $^{1}$ de ubicación retropúbica. Su eficacia a largo plazo está probada con publicaciones de seguimiento mayores de 7 años ${ }^{12,13}$ y de hecho hoy es el "gold estándar" para la cirugía de la incontinencia urinaria de esfuerzo. Como consecuencia de las potenciales lesiones graves que se pueden producir con esta técnica y la posible lesión vesical al paso de las agujas que obliga a realizar una cistoscopia, en el año 2001 (Delorme) $^{2}$ y 2003 (Leval) ${ }^{3}$ aparecen la segunda generación de cintas con abordaje transobturador con menor porcentaje de lesiones graves y obviando la necesidad de cistoscopia.

En el año 2006 (TVT secur) y en el 2007 (MiniArc y Needeleless) surgen la tercera generación de cintas, en las que su longitud reduce en 2/3 pasando de 20$21 \mathrm{~cm}$ a 8-8,5 cm. Estas cintas tienen como ventajas:

- Su colocación en dos posiciones posibles: una como U (similar a la TVT) o como en V (hamaca TOT/TVT-O).

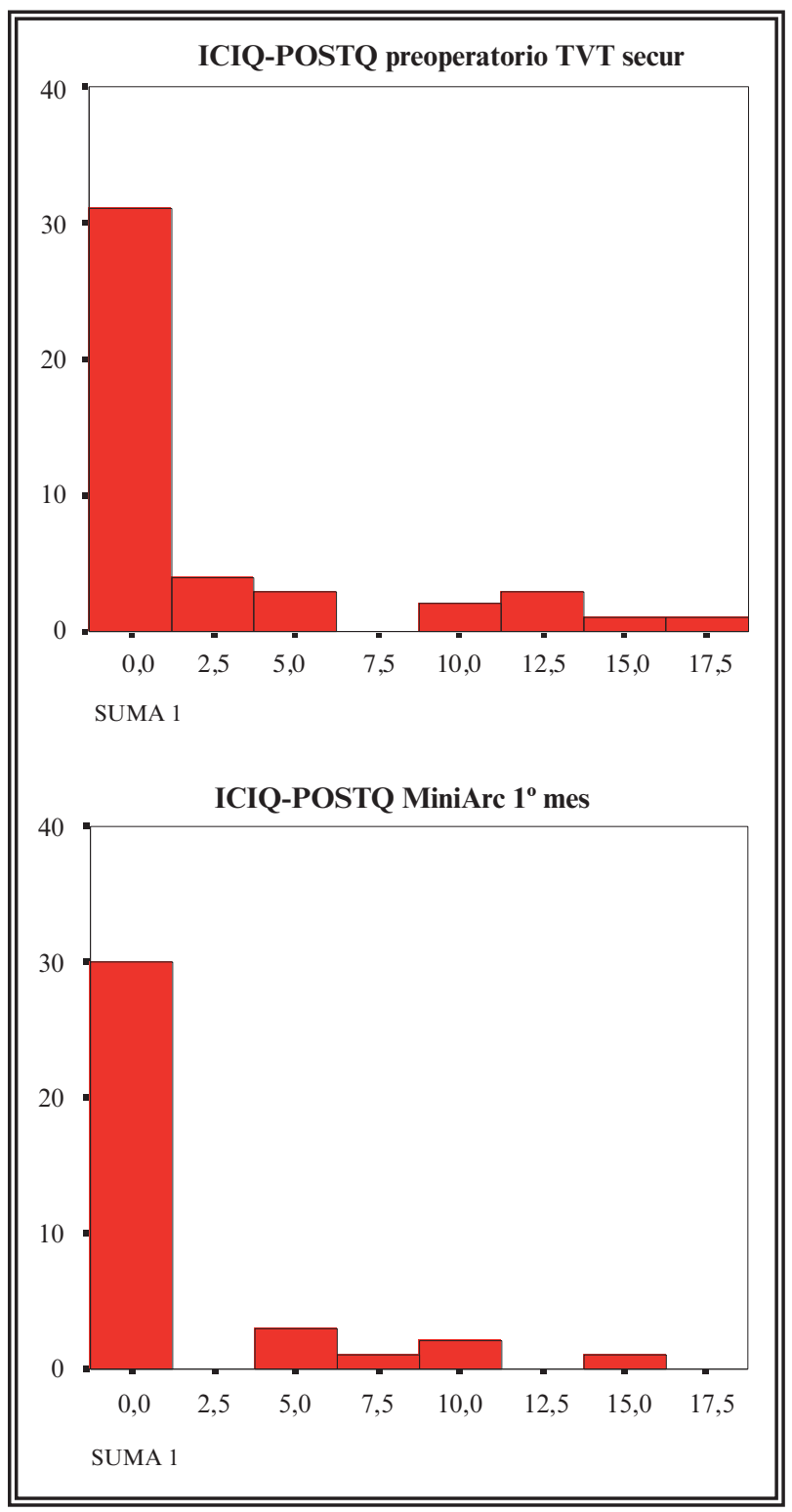

FIGURA 3. ICIQ postoperatorio $1^{\circ}$ mes.

- Una menor penetración de tejidos que sus antecesoras.

- Un menor número de complicaciones, comparada con nuestra serie de TVT y TVT-O ${ }^{6,7}$.

- Un menor dolor postoperatorio ${ }^{17}$.

- Y la posibilidad de realizar la cirugía con anestesia local y en régimen ambulatorio.

En un estudio publicado por Rezapour et $\mathrm{al}^{4}$. en el 2006, se demuestra que a pesar de reducir el tamaño de la cinta se obtiene el mismo efecto a nivel de la uretra media manteniendo la fuerza 10 veces superior a la tensión sostenida por la fascia y prologándose estos efectos físicos sobre la uretra en el tiempo. 


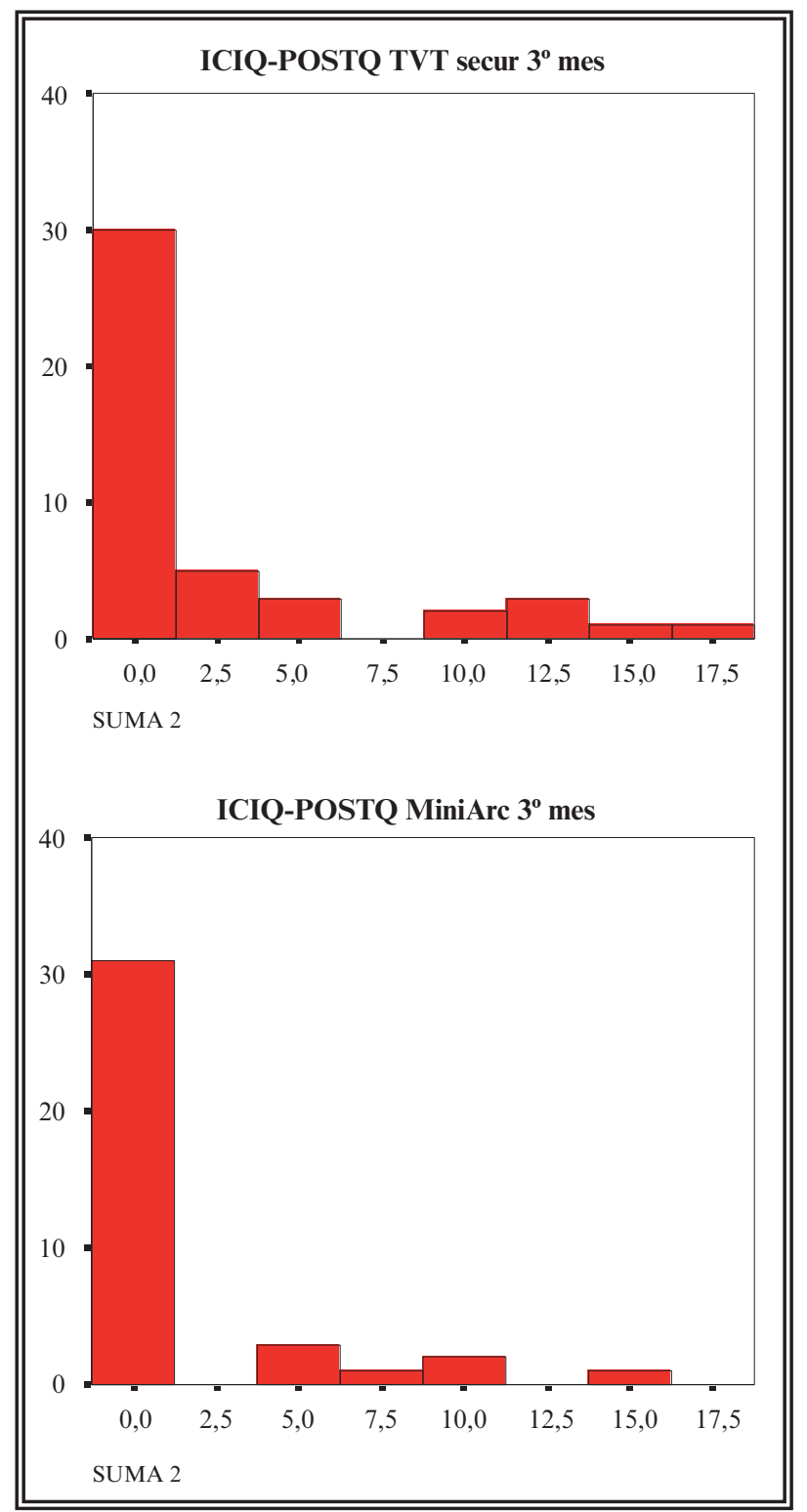

FIGURA 4. ICIQ postquirurgico $3^{\circ}$ mes.

Los resultados que obtenemos en nuestra serie de TVT secur son equiparables a los publicados y presentados en diferentes congresos ${ }^{5,8-11}$, con la diferencia de que nuestro seguimiento es algo mayor y respecto a la serie de MiniArc ocurre prácticamente lo mismo teniendo en cuenta que sólo existe una sola publicación patrocinada por $\mathrm{AMS}^{16}$.

Si comparamos los resultados en cuanto a curación objetiva, observamos como los resultados con estas cintas son sensiblemente inferiores a los obtenidos con sus precedecesoras ${ }^{6,7}$.

En cuanto a las complicaciones quirúrgicas, la perforación vesical es anecdótica (1 paciente) y las

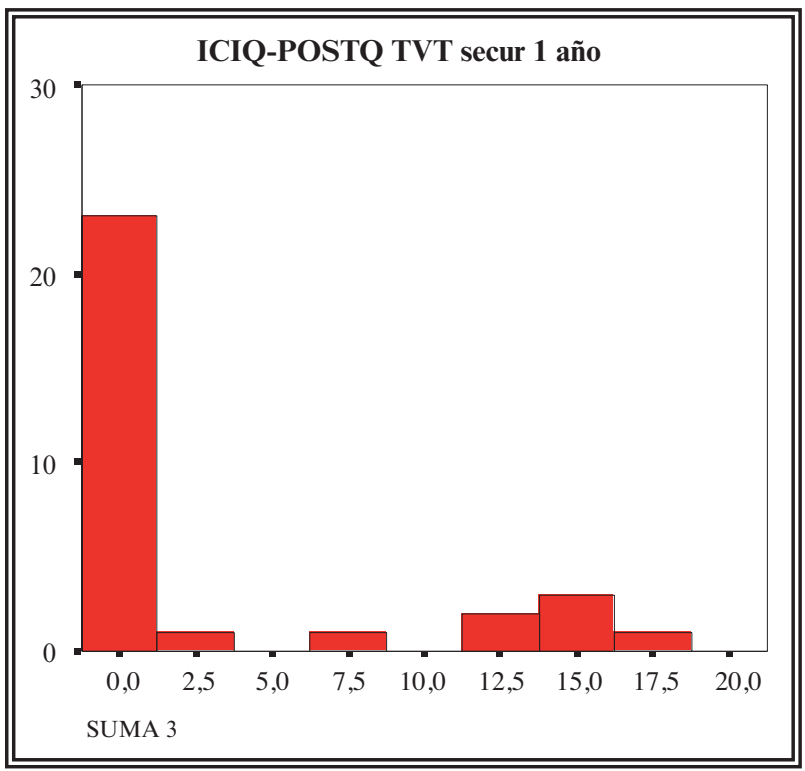

FIGURA 5. ICICQ-SF TVT secur al año.

complicaciones postoperatorias son equiparables a las publicadas excepto en la aparición de urgencia de novo, mayor en nuestra serie de TVT secur $(9,8 \%$ vs $4,2 \%)^{8-12,15}$.

\section{CONCLUSIÓN}

Estas minicintas son una nueva herramienta para el tratamiento de la incontinencia de esfuerzo con una claras ventajas respecto a la TVT y TOT/TVT-O, menor riesgo de complicaciones graves, menor dolor postoperatorio y fundamentalmente la posibilidad de realizar la cirugía con anestesia local consiguiendo dar la tensión adecuada a la cinta in situ, pero debe demostrar sus resultados a largo plazo teniendo como referencia al "gold stándar" de la TVT.

\section{REFERENCIAS}

1. Ulmsten U, Henriksson L, Johnson P, Varhos G. Ambulatory surgical procedure under local anesthesia for treatment of femaleurinary incontinence. Int Urogynecol J Pelvic Floor Dusfunct. 1996;7(2):81-85.

2. Delorme E. Transobturator urethral suspension:miniinvasive procedure in the treatment of stress urinary incontinence in women. Prog Urol. 2001;11(6):1306-1313. 3. De Leval J. Novel surgical technique for treatment of female stress urinary incontinence:transobturator vaginal tape inside-out. Eur Urol. 2003; 44(6):724-730.

4. Rezapour M, Novara G, Meier PA, Holste J, Landgrebe S, Artibani W. A 3 month preclinical trial to asses the performance of a new TVT-like mesh (TVTx) in a sheep model.Int Urogynecol J Pelvic Floor Dysfunct. 2007;18(2):183-187.

5. Vicente Sola D, Jack Pardo, Paolo Ricci. Tercera generación en cirugía minimamente invasiva para la corrección de la incontinencia de orina de esfuerzo: TVT secur. Rev Chil Obstet ginecol. 2007;72(5):304-309. 
6. Hualde A, Jimenez Calvo $\mathrm{J}$ et al. Nuestra experiencia a cinco años y medio en TVT. Actas urol esp 2006 30(2):181-185.

7. Jimenez Calvo J,Hualde Alfaro A et al. TOT en el tratamiento de la incontinencia urinaria de esfuerzo:nustra experiencia comparándola con la TVT. Actas Urol Esp. 2007;31(10) 11341140.

8. Karram M, Lucente V et al. An evaluation of the gynecare TVT secur system for the treatment of stress urinary incontinence. Int Urogynecol J 2007; 18(suppl1) S3.

9. Marsh FA, Assassa P. An audit of the introduction of tvt secur in clinical practice. Int Urogynecol J 2007;18(suppl1) S26.

10. Martan A, Masata J Svabick. Initial experience with tvt-secur system procedure and reason for persistent stress urinary incontinence. Int Urogynecol J 2007;18(suppl1) S26.

11. Saltz SM,Haff RE,Lucente V. Short term assessment of patients undergoing the new tension free vaginal tape:secur procedure for treatment of stress urinary incontinence. Int Urogynecol $\mathrm{J}$ 2007;18(supp11) S27.

12. Deffieux X,Donnadieu A, Porcher R.Long term results of tension free vaginal tape for female urinary incontinence.Folow up over 6 years. International Journal of Urology 2007;14(6):521526.

13. Chene G, Amblard J, Tardieu A et al. Long term results of tension free vaginal tape for treatment of female urinary stress incontinence. Eur J obstet gynecol reprod biol 2007;134(1):87-94.
14. Espuña M,Rebollo P, Puig M. Validación de la versión española de la international consultation on incontinence questionnarie short form. Un cuestionario para evaluar la incontinencia urinaria. Med Clin. 2004;122(8):288-292.

15. Debodinance P, Lagrange E, Amblard J et al. TVT securtrade mark: More and more minimal invasive.Preliminary prospective study on 110 cases. J Gynecol obstet boil Reprod. 2008 Mar; (14).

16. $\mathrm{R}$ Moore et al. Retrospective review of early experience using the AMS Mini Arc Single Incision Sling System to treat stress urinary incontinence in women. The Journal of Minimally Invasive Gynecology 2007;(14): S129-130.

17. Sola Dalenz V, Ricci Arriola P, Pardo Schanz J. Corrección quirúrgica d ela incontinencia de orina de esfuerzo, con cinta sintética sub-medio-uretral de tercera generación: TVT-Secur. Actas Urol Esp. 2008;32(5):522-529.

Correspondencia autor: Dr. J. Jiménez Calvo Servicio de Urología. Hospital Virgen del Camino Irunlarrea, 4 - 31008 Pamplona (Navarra)

Tel.: 948429400

E-mail autor: auvc03@yahoo.es

Información artículo: Original - Incontinencia

Trabajo recibido: mayo 2008

Trabajo aceptado: julio 2008 